Article

\title{
Hydration of terminal alkynes catalyzed by a water-soluble salen-Co(III) complex
}

\author{
Shoufeng Wang a,b, Chengxia Miao ${ }^{b}$, Wenfang Wang ${ }^{b}$, Ziqiang Lei ${ }^{\mathrm{a}, *}$, Wei Sun ${ }^{\mathrm{b}, \#}$ \\ a Laboratory of Eco-Environment-Related Polymer Materials of Ministry of Education, Key Laboratory of Polymer Materials of Gansu Province, College of \\ Chemistry and Chemical Engineering, Northwest Normal University, Lanzhou 730070, Gansu, China \\ ${ }^{\mathrm{b}}$ State Key Laboratory for Oxo Synthesis and Selective Oxidation, Lanzhou Institute of Chemical Physics, Chinese Academy of Sciences, Lanzhou 730000, \\ Gansu, China
}

\section{A R T I C L E I N F O}

\section{Article history:}

Received 20 March 2014

Accepted 11 April 2014

Published 20 October 2014

\section{Keywords:}

Hydration

Alkynes

Water-solubility

Cobalt

Salen complex

\section{A B S T R A C T}

A water-soluble salen-Co(III) complex was studied as catalyst for hydration of terminal alkynes to methyl ketones in the presence of $\mathrm{H}_{2} \mathrm{SO}_{4}$ as a co-catalyst. The products were obtained with excellent yields using relatively low catalyst loadings and a simple protocol. Notably, the products were easily separated from the catalyst after reaction by extraction, and the catalyst could be recovered and reused with only a slight loss of activity.

(C) 2014, Dalian Institute of Chemical Physics, Chinese Academy of Sciences. Published by Elsevier B.V. All rights reserved.

\section{Introduction}

Addition of water to alkynes is very important in organic synthesis because, in principle, addition reactions can be performed with $100 \%$ atom efficiency. This means there is no waste formation and the reactions fulfill the requirements of green chemistry. The traditional method for alkyne hydration involves catalysis with a $\mathrm{Hg}(\mathrm{II})$ salt, and selectivity follows Markovnikov's rule yield methyl ketones [1-7]. This method is still in use today because it is reliable. However, over the past 30 years, metal catalysts with low toxicity and high activity, such as Pt [8] Fe [9,10], Pd [11], Ir [12], Ag [13-15], Au [16-18], Ru [19-24] have been investigated to replace $\mathrm{Hg}(\mathrm{II})$. In general, the more active and selective of the available catalysts for alkyne hydra- tion are based on expensive transition metals. Separation of products from these catalytic systems requires tedious procedures. Very recently, Naka group [25] developed a novel method for hydration of terminal alkynes catalyzed by a recyclable, water-soluble porphyrin-Co(III) complex. Compared with heterogeneous analogues, soluble homogeneous supports provide increased solvent compatibility and accelerated reaction rates.

Inspired by pioneering research in this area and the continued interest in catalysis with salen metal complexes [26-30], we investigated a water-soluble salen-Co(III) complex as a catalyst for hydration of terminal alkynes. This complex conveniently catalyzed hydration of terminal alkynes in good yields. After the reaction, the products were easily separated by extraction with an organic solvent, and the catalyst could be re-

\footnotetext{
* Corresponding author. E-mail: leiziqiang@hotmail.com

\# Corresponding author. Fax: +86-931-8277088; E-mail: wsun@licp.cas.cn

This work was supported by the National Natural Science Foundation of China $(21103207,21133011)$.

DOI: 10.1016/S1872-2067(14)60105-4 | http://www.sciencedirect.com/science/journal/18722067 | Chin. J. Catal., Vol. 35, No. 10, October 2014
} 
used three times.

\section{Experimental}

\subsection{General}

Phenylacetylene derivatives and other commercially available chemicals were obtained from Alfa Aesar, Sigma-Aldrich, J\&K Scientific Ltd, or Tokyo Chemical Industry Co., Ltd and used as received without pretreatment unless otherwise stated. Salen ligands and salen-Co(III)-OAc complexes C1 and C2 were prepared according to reported methods [31]. The substituted sulfonatosalen-Co(III) complex C3 was synthesized by condensation of the 1,2-ethylenediamine with 5-sulfonatosalicylaldehyde and sequential addition of cobalt acetate in good yield (see Scheme 1) [31,32]. 4-Ethynylbiphenyl, 4-ethynyl1,2-dimethoxybenzene, and 1-ethynyl-naphthalene were prepared according to literature procedures [33].

\subsection{General procedure for hydration of terminal alkynes}

The $10 \mathrm{~mL}$ schlenk tube was charged with phenylacetylene $(0.5 \mathrm{mmol}, 51 \mathrm{mg})$, methanol $(0.625 \mathrm{~mL})$, catalyst $(10 \mu \mathrm{mol}$, $2.0 \%)$, and then $\mathrm{H}_{2} \mathrm{SO}_{4}(10 \mu \mathrm{mol}, 2.0 \%)$ dissolved in $\mathrm{H}_{2} \mathrm{O}(2.2$ mmol, $0.04 \mathrm{~mL}$ ). The mixture was heated to $80{ }^{\circ} \mathrm{C}$ and at it for $20 \mathrm{~h}$ in a closed tube with a magnetic stirring bar. The progress of the reaction was monitored using TLC and GC-MS. After the reaction, the mixture was cooled to room temperature, and $\mathrm{CH}_{2} \mathrm{Cl}_{2}(5 \mathrm{~mL})$ and water $(5 \mathrm{~mL})$ were added to the mixture. The aqueous and organic layers were separated, and the aqueous phase was extracted with $\mathrm{CH}_{2} \mathrm{Cl}_{2}(5 \mathrm{~mL} \times 3)$. The combined organic extracts were washed with a saturated $\mathrm{NaCl}$ solution, dried over $\mathrm{Na}_{2} \mathrm{SO}_{4}$, and concentrated under reduced pressure. Then the product acetophenone was abtained.

\section{Results and discussion}

\subsection{Optimization of reaction conditions}

As we previously reported, the salen-Co(III) complex $\mathbf{C 1}$ and C2 could catalyze the hydration of terminal alkynes in the presence of $\mathrm{H}_{2} \mathrm{SO}_{4}$ (Table 1, entries 1 and 2) [26]. Taking into account the problem of product isolation, a water-soluble
Table 1

Screening of reaction conditions for the hydration of phenylacetylene.

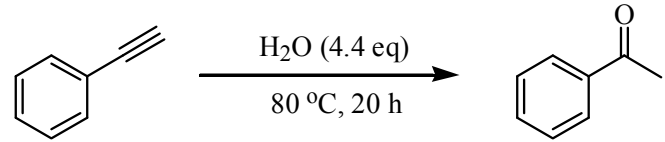

\begin{tabular}{lcccc}
\multicolumn{2}{c}{ 1a } & & \multicolumn{2}{c}{ 2a } \\
\hline Entry & Catalyst & Solvent & Acid (\%) & Yield ${ }^{\mathrm{a}}(\%)$ \\
\hline 1 & $\mathbf{C} 1$ & $\mathrm{CH}_{3} \mathrm{OH}$ & $\mathrm{H}_{2} \mathrm{SO}_{4}(2.0)$ & 99 \\
2 & $\mathbf{C} 2$ & $\mathrm{CH}_{3} \mathrm{OH}$ & $\mathrm{H}_{2} \mathrm{SO}_{4}(2.0)$ & 99 \\
3 & $\mathbf{C 3}$ & $\mathrm{CH}_{3} \mathrm{OH}$ & $\mathrm{H}_{2} \mathrm{SO}_{4}(2.0)$ & 99 \\
4 & $\mathbf{C 3}$ & $\mathrm{CH}_{3} \mathrm{OH}$ & $\mathrm{HCl}(4.0)$ & 94 \\
5 & $\mathbf{C 3}$ & $\mathrm{CH}_{3} \mathrm{OH}$ & $\mathrm{CF}_{3} \mathrm{SO}_{3} \mathrm{H}(4.0)$ & 75 \\
6 & $\mathbf{C 3}$ & $\mathrm{CH}_{3} \mathrm{OH}$ & $\mathrm{HNTF}_{2}(4.0)$ & 45 \\
7 & $\mathbf{C 3}$ & $\mathrm{CH}_{3} \mathrm{OH}$ & $\mathrm{H}_{2} \mathrm{SO}_{4}(2.0)$ & $78^{\mathrm{b}}$ \\
8 & $\mathbf{C 3}$ & $\mathrm{H}_{2} \mathrm{O}$ & $\mathrm{H}_{2} \mathrm{SO}_{4}(2.0)$ & 5 \\
9 & $\mathbf{C 3}$ & $\mathrm{CH}_{3} \mathrm{OH}$ & $\mathrm{H}_{2} \mathrm{SO}_{4}(2.0)$ & $91^{\mathrm{c}}, 89^{\mathrm{d}}, 83^{\mathrm{e}}$ \\
10 & $\mathbf{C 3}$ & $\mathrm{CH}_{3} \mathrm{OH}$ & $\mathrm{H}_{2} \mathrm{SO}_{4}(2.0)$ & $96^{\mathrm{f}}$ \\
\hline
\end{tabular}

Reaction conditions: phenylacetylene $0.5 \mathrm{mmol}$, catalyst $10 \mu \mathrm{mol}$ (2.0\%), $\mathrm{H}_{2} \mathrm{O} 4.4$ eq. $(40 \mu \mathrm{L})$, solvent $0.625 \mathrm{~mL}$, [Alkyne $]_{0}=0.8 \mathrm{~mol} / \mathrm{L}, 80$ ${ }^{\circ} \mathrm{C}, 20 \mathrm{~h}$.

a The yield was determined by GC with nitrobenzene as an internal standard.

${ }^{b}$ The reaction was carried out under an argon atmosphere.

c First run, isolated yield.

${ }^{\mathrm{d}}$ Second run, isolated yield.

e Third run, isolated yield.

fPhenylacetylene $10.0 \mathrm{mmol}$, isolated yield.

salen-Co(III) complex C3 was developed as an ideal catalyst for the hydration of terminal alkynes. As expected, the hydration of phenyl acetylene to the corresponding acetophenone proceeded uneventfully in the presence of the water-soluble salen-Co(III) complex $\mathbf{C 3}(2.0 \%)$ and $2.0 \% \mathrm{H}_{2} \mathrm{SO}_{4}$ (Table 1, entriey 3). Preliminary results indicated that including an acid as a co-catalyst greatly influenced the hydration, but among the acids tested, only $\mathrm{HCl}$ provided a comparable yield to $\mathrm{H}_{2} \mathrm{SO}_{4}$ (Table 1, entries 3-6). As Naka group reported [25], alkyne hydration was even faster in the presence of $\mathrm{HNTf}_{2}$. However, for the water-soluble salen-Co(III) complex, $\mathrm{HNTf}_{2}$ could not promote the hydration efficiently. In addition, when the reaction was carried out under an Ar atmosphere, the ketone yield decreased (Table 1, entry 7). As reported by Naka group [25], methanol was an excellent solvent for the hydration of terminal alkynes catalyzed by water-soluble porphyrin-Co(III) complexes (Table 1, entry 3 vs 8 ).

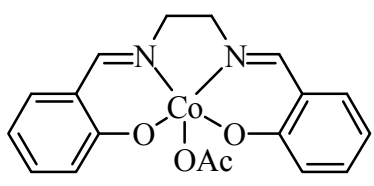

C1

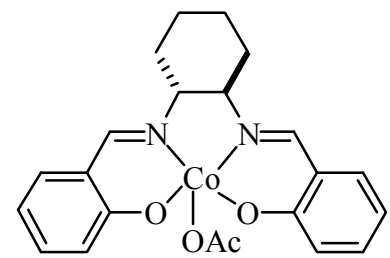

C2

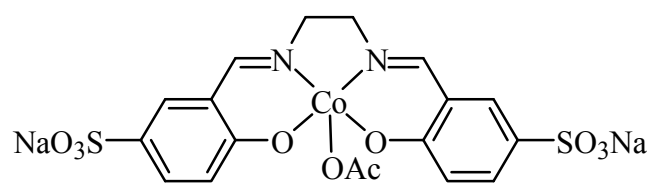

C3

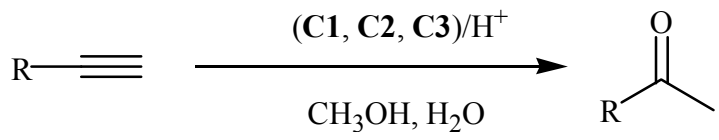

Scheme 1. Hydration of alkynes catalyzed by salen-Co(III) complexes. 


\subsection{Catalyst recyclability and a gram-scale hydration}

An important feature of this substituted sulfonato-salen-Co(III) complex-catalyzed hydration of terminal alkynes is the facile separation of the catalyst and the product. After every hydration reaction, the solution $\mathrm{pH}$ was adjusted to 7 with a $\mathrm{NaOH}$ solution, the cooled mixture was partitioned between water and $n$-hexane or dichloromethane, and the organic layer was separated. The water-soluble Co complex in the aqueous layer was recovered by evaporating water, and the complex was then reused for further reactions. The catalyst $\mathbf{C} 3$ could be reused three times with only a slight loss of activity (Table 1, entry 9). To evaluate the scalability of the reaction, a gram-scale hydration of phenylacetylene $(10 \mathrm{mmol})$ was carried out under the optimal conditions (Table 1, entry 10). The hydration proceeded smoothly with acetophenone obtained in $96 \%$ yield.

\subsection{Substrate scope}

To explore the synthetic utility of the catalyst, we investigated the substrate scope under the optimized conditions. A series of aromatic alkynes with different substituent groups on the phenyl ring, heteroaromatic alkynes, and aliphatic alkynes were subjected to this hydration process with catalyst C3 (Table 2). The results clearly demonstrated that the reaction was influenced by the electronic effect. Aromatic alkyne derivates with electron-donating substitutents, such as methyl, $t$-butyl, phenyl, and methoxy, reacted smoothly to afford the desired methyl ketones in high yields (Table 2, entries 2-8). However, those with electron-withdrawing substituents, such as $-\mathrm{F},-\mathrm{Cl}$, or $-\mathrm{Br}$ groups, showed decreased conversion. Interestingly, when the fraction of $\mathrm{H}_{2} \mathrm{SO}_{4}$ was increased from $2 \%$ to $10 \%$, the effectiveness of the cobalt catalyst for these substrates also increased (Table 2, entries 9-11). By contrast, for phenylacetylene with a strong electron-withdrawing group (-CN), no hydration took place (Table 2, entry 12). Heterocyclic aromatic alkynes were also efficiently hydrated in this reaction (Table 2, entries 14 and 15). For example, the reaction for 4-ethynylpyridine proceeded smoothly under typical conditions, affording 4-acetylpyridine in 98\% yield (Table 2, entry 14). Aliphatic alkyne such as 1-decyne was less active than aromatic alkynes, with only $37 \%$ yield (Table 2 , entry 16 ). The hydration product was not obtained with the simple internal alkyne 3-phenyl-1- propyne (Table 2, entry 17).

\subsection{Mechanism}

To gain insight into the mechanism of Co-catalyzed hydration of alkynes, quadrupole-time-of-flight-mass spectrometry was used to investigate the hydration of terminal alkynes. Salen-Co(III) complex C1 was used as the catalyst. Phenylacetylene $(0.5 \mathrm{mmol})$ and catalyst $\mathbf{C 1}(2 \%)$ in methanol $(0.6 \mathrm{~mL})$ were stirred at $50{ }^{\circ} \mathrm{C}$ for $1 \mathrm{~h}$. After this reaction, peaks appeared at $\mathrm{Mr} / z=325.0379$ and 449.0652, and could be assigned to [salen-Co(III) $]^{+}$and [salen-Co(III)-phenylacetylene+Na] ${ }^{+}$(Fig. 1), respectively. These results indicate that a cobalt(III) alkyne complex intermediate was formed between the salen-Co(III)
Table 2

Substrate scope for alkyne hydration catalyzed by C3.

Entry

Reaction conditions: substrate $0.5 \mathrm{mmol}$, catalyst $10 \mu \mathrm{mol}(2.0 \%)$, $\mathrm{H}_{2} \mathrm{SO}_{4} 10 \mu \mathrm{mol}(2.0 \%), \mathrm{H}_{2} \mathrm{O} 4.4$ eq. $\left(40 \mu \mathrm{L}\right.$ ), $\mathrm{CH}_{3} \mathrm{OH} 0.625 \mathrm{~mL}$ ([Alkyne] $=0.8 \mathrm{~mol} / \mathrm{L}), 80^{\circ} \mathrm{C}, 20 \mathrm{~h}$.

${ }^{a}$ Isolated yield.

${ }^{\mathrm{b}} \mathrm{H}_{2} \mathrm{SO}_{4} 50 \mu \mathrm{mol}(10 \%)$.

c The yield was determined by GC.

${ }^{\mathrm{d}} 50^{\circ} \mathrm{C}$ for $20 \mathrm{~h}$, the yield was determined by GC.

complex and phenylacetylene. Taking into account our previous work and other reports [30,34], the Co(III) alkyne complex intermediate is most likely then attacked by methanol to generate enol ether in the presence of the acid co-catalyst (Scheme 2(1)). The enol ether will then be transformed into the desired ketone in the presence of the acid co-catalyst and water under acidic conditions (Scheme 2(2)).

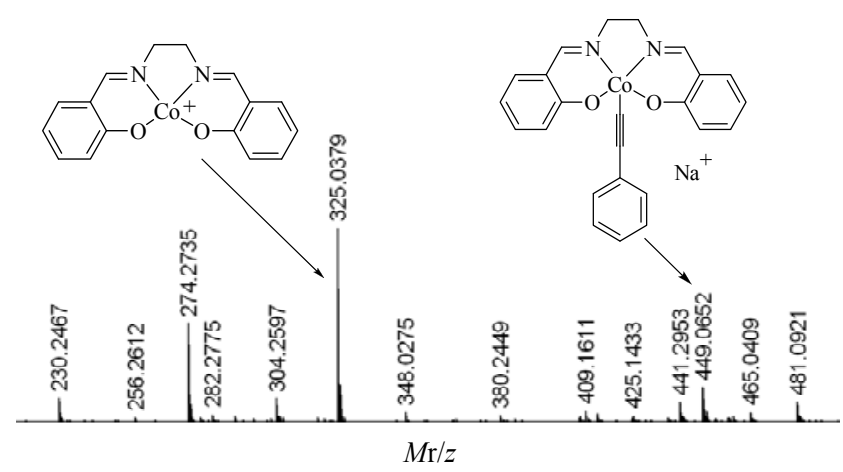

Fig. 1. HRMS spectra of the reactants. Phenylacetylene $0.5 \mathrm{mmol}$, C1 $2 \%$, methanol $0.6 \mathrm{~mL}, 50^{\circ} \mathrm{C}, 1 \mathrm{~h}$. 
(1)

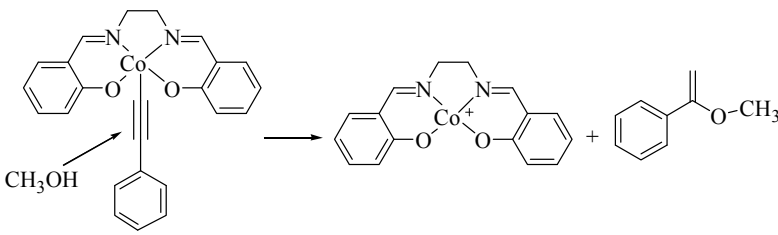

(2)

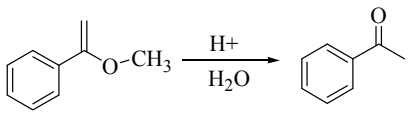

Scheme 2. Proposed mechanism for salen-Co(III) catalyzed hydration.

\section{Conclusions}

In conclusion, a water-soluble salen-Co(III) complex was developed for the hydration of terminal alkynes using $\mathrm{H}_{2} \mathrm{SO}_{4}$ as a co-catalyst. This catalyst transformed various alkynes to methyl ketones in good to excellent yields. The products could be isolated by simple extraction from the reaction mixture, which avoided column chromatography. The catalyst could be reused three times with only a slight loss of activity for the reaction. Overall, this water-soluble salen-Co(III) complex catalyst makes the hydration of terminal alkynes very practical. Applications of this water-soluble salen-Co(III) complex to other reactions and further experimental are ongoing in our laboratory.

\section{References}

[1] Song F, Liu Y M, Wang L L, Zhang H J, He M Y, Wu P. Appl Catal A, 2007, 327: 22

[2] Hintermann L, Labonne A. Synthesis, 2007, 1121

[3] Beller M, Seayad J, Tillack A, Jiao H J. Angew Chem Int Ed, 2004, 43: 3368

[4] Alonso F, Beletskaya I P, Yus M. Chem Rev, 2004, 104: 3079

[5] Kutscheroff M. Ber Dtsch Chem Ges, 1884, 17: 13

[6] Kutscheroff M G. Ber Dtsch Chem Ges, 1909, 42: 2759

[7] Killian D B, Hennion G F, Nieuwland J A. J Am Chem Soc,1936, 58: 80

[8] Hiscox W, Jennings P W. Organometallics, 1990, 9: 1997

[9] Wu X F, Bezier D, Darcel C. Adv Synth Catal, 2009, 351: 367
[10] Cabrero-Antonino J R, Leyva-Pérez A, Corma A. Chem Eur J, 2012, 18: 11107

[11] Fukuda Y, Shiragami H, Utimoto K, Nozaki H.J Org Chem, 1991, 56: 5816

[12] Hirabayashi T, Okimoto Y, Saito A, Morita M, Sakaguchi S, Ishii Y. Tetrahedron, 2006, 62: 2231

[13] Thuong M B T, Mann A, Wagner A. Chem Commun, 2012, 48: 434

[14] Saha S, Sarbajna A, Bera J K. Tetrahedron Lett, 2014, 55: 1444

[15] Rao K T V, Prasad P S S, Lingaiah N. Green Chem, 2012, 14: 1507

[16] Corma A, Ruiz V R, Leyva-Prez A, Sabater M J. Adv Synth Catal, 2010, 352: 1701

[17] Marion N, Ramón R S, Nolan S P. J Am Chem Soc, 2009, 131: 448

[18] Mizushima E, Sato K, Hayashi T, Tanaka M. Angew Chem Int Ed, 2002, 41: 4563

[19] Boeck F, Kribber T, Xiao L, Hintermann L. J Am Chem Soc, 2011, 133: 8138

[20] Chevallier F, Breit B. Angew Chem Int Ed, 2006, 45: 1599

[21] Grotjahn D B, Lev D A. J Am Chem Soc, 2004, 126: 12232

[22] Li L, Herzon S B. J Am Chem Soc, 2012, 134: 17376

[23] Li L, Herzon S B. Nat Chem, 2014, 6: 22

[24] Zeng M S, Li L, Herzon S B. J Am Chem Soc, 2014, 136: 7058

[25] Tachinami T, Nishimura T, Ushimaru R, Noyori R, Naka H. J Am Chem Soc, 2013, 135: 50

[26] Xiong D L, Wu M, Wang S F, Li F W, Xia C G, Sun W. Tetrahedron: Asymmetry, 2010, 21: 374

[27] Lv C W, Cheng Q G, Xu D Q, Wang S F, Xia C G, Sun W. Eur J Org Chem, 2011, 3407

[28] Xiong D L, Hu X X, Wang S F, Miao C X, Xia C G, Sun W. Eur J Org Chem, 2011, 4289

[29] Xu D Q, Wang S F, Shen Z Q, Xia C G, Sun W. Org Biomol Chem, 2012, 10: 2730

[30] Wang S F, Miao C X, Wang W F, Lei Z Q, Sun W. ChemCatChem, 2014, 6: 1612

[31] Schaus S E, Brandes B D, Larrow J F, Tokunaga M, Hansen K B, Gould A E, Furrow M E, Jacobsen E N. J Am Chem Soc, 2002, 124: 1307

[32] Wu Z Q, Zhou Li, Jiang Z Q, Wu D, Li Z K, Zhou X G. Eur J Org Chem, 2010, 4971

[33] Zhou N Z, Wang L, Thompson D W, Zhao Y M. Tetrahedron, 2011, 67: 125

[34] Teles J H, Brode S, Chabanas M. Angew Chem Int Ed, 1998, 37: 1415

\section{Graphical Abstract}

Chin. J. Catal., 2014, 35: 1695-1700 doi: 10.1016/S1872-2067(14)60105-4

\section{Hydration of terminal alkynes catalyzed by water-soluble salen-Co(III) complex}

Shoufeng Wang, Chengxia Miao, Wenfang Wang, Ziqiang Lei *, Wei Sun*

Northwest Normal University; Lanzhou Institute of Chemical Physics, Chinese Academy of Sciences

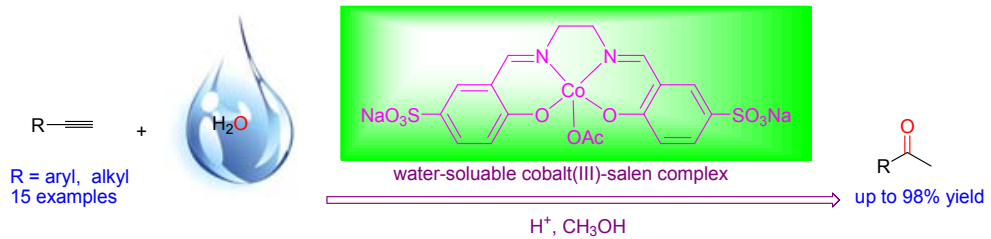

A water-soluble sulfonato-Co(salen) complex was studied as a catalyst for hydration of terminal alkynes to give methyl ketones in high yields. The product can be easily separated from the catalyst by extraction using water and an organic solvent, and the catalyst can be recovered and reused. 


\title{
水溶性salen-Co(III)配合物催化的端炔水合反应
}

\author{
王寿峰 ${ }^{\mathrm{a}, \mathrm{b}}$ ，苗成霞，王文芳 ${ }^{\mathrm{b}}$, 雷自强, ${ }^{\mathrm{a}}$ ，孙 伟 ${ }^{\mathrm{b}, \#}$ \\ ${ }^{\mathrm{a}}$ 西北师范大学化学化工学院, 生态环境相关高分子材料教育部重点实验室, 甘肃省高分子材料重点实验室, 甘肃兰州730070 \\ ${ }^{\mathrm{b}}$ 中国科学院兰州化学物理研究所, 羰基合成与选择氧化国家重点实验室, 甘肃兰州730000
}

摘要: 报道了一种用于端炔水合反应的水溶性salen-Co(III)配合物催化剂, 在使用硫酸作为共催化剂的条件下能高效得到产物甲 基酮. 该催化剂用量少, 反应结束后可利用简单的萃取实现产物与催化剂分离, 简化了后处理过程. 此外, 催化剂还可回收重复使 用, 但催化剂活性会略有下降.

关键词: 水合反应; 炔烃; 水溶性; 钴; 希夫碱配合物

收稿日期: 2014-03-20. 接受日期: 2014-04-11. 出版日期: 2014-10-20.

*通讯联系人. 电子信箱: leiziqiang@hotmail.com

\#通讯联系人. 传真: (0931) 8277088; 电子信箱: wsun@licp.cas.cn

基金来源：国家自然科学基金(21103207, 21133011).

本文的英文电子版由Elsevier出版社在ScienceDirect上出版(http://www.sciencedirect.com/science/journal/18722067).

\section{1. 前言}

炔烃水合反应是合成化学中重要的官能团转换方法 之一. 从合成的角度看, 该反应的原子经济性达 $100 \%$, 符 合绿色化学和可持续发展的要求. 常用的方法是端炔在 $\mathrm{Hg}(\mathrm{II})$ 盐和过量的硫酸存在下水合反应生成符合马尔科夫 加成规则的甲基酮, $\mathrm{Hg}$ (II)盐由于其稳定的催化性能直到 现在还在使用, 但造成严重的环境污染 ${ }^{[1-7]}$. 为了克服这些 缺陷, 在过去的 30 年里, 科研工作者们广泛研究了非 $\mathrm{Hg}$ 的 金属盐或配合物, 如 $\mathrm{Pt}^{[8]}, \mathrm{Fe}^{[9,10]}, \mathrm{Pd}^{[11]}, \mathrm{Ir}^{[12]}, \mathrm{Ag}^{[13-15]}$, $\mathrm{Au}^{[16-18]}, \mathrm{Ru}^{[19-24]}$ 等, 期望找到比 $\mathrm{Hg}$ (II)毒性小且活性高的 催化剂. 一般来说, 高活性的催化剂集中在贵金属上且产 物和催化剂的分离过程比较复杂. 最近, Naka课题组 ${ }^{[25]}$ 取 得了突破性的进展, 他们使用一种可回收的水溶性卟啉钴 催化剂用于端炔的水合反应. 相对于常用的均相催化剂负 载方法而言, 该催化剂具有更好的溶解性和催化活性.

基于上述文献的启发和在 salen配体及其衍生物的 合成与应用等方面的经验 ${ }^{[26-30]}$, 我们考虑使用合成路线 相对简单, 廉价易得的水溶性salen-Co(III)配合物催化剂 用于炔烃的水合反应中. 结果表明, 该催化剂也能够高 效地催化炔烃水合反应. 在该催化体系中, 产物可以用 萃取的方法分离出来, 催化剂可以重复使用 3 次.

\section{2. 实验部分}

\section{1. 试剂与催化剂}

苯乙炔、取代苯乙炔和其他有机试剂购买自Alfa Aesar公司, Aldrich公司, 百灵威公司或东京化成公司, 使用前未经纯化. Salen配体、salen-Co(III)-OAc配合物
C1 和 C2 根据文献[31]合成. 磺酸盐取代的salen-Co(III) 配合物 C3 参照文献[31,32]合成, 即先由乙二胺和5-磺酸 盐水杨醛缩合, 再和醋酸钴配合然后空气氧化得到(见 图式1). 4-乙炔基联苯、4-乙炔基-1,2-二甲氧基苯和1-乙 炔基荎参照文献[33]合成.

\section{2. 炔烃水合反应的一般过程}

在 $10 \mathrm{~mL}$ schlenk反应管中加入底物苯乙炔 $0.5 \mathrm{mmol}$ (51 mg), 甲醇 $0.625 \mathrm{~mL}$, 催化剂 $(10 \mu \mathrm{mol}, 2.0 \%)$, 然后 将 $\mathrm{H}_{2} \mathrm{SO}_{4}(10 \mu \mathrm{mol}, 2.0 \%)$ 溶解在水 $(2.2 \mathrm{mmol}, 0.04$ $\mathrm{mL})$ 中一起加入, 密封, 磁力搅拌, 在 $80^{\circ} \mathrm{C} 反$ 应 $20 \mathrm{~h}$. 反应过程由 TLC 和 GC-MS监测. 反应完毕冷却到室 温, 加入 $5 \mathrm{~mL}$ 水和 $5 \mathrm{~mL} \mathrm{CH}_{2} \mathrm{Cl}_{2}$, 混合物分层, 水相用 $\mathrm{CH}_{2} \mathrm{Cl}_{2}$ 多次萃取 $(5 \mathrm{~mL} \times 3)$, 合并有机相, 水洗, 饱和 食盐水洗, 无水硫酸钠干燥, 减压除溶剂, 得到苯乙 酮.

\section{3. 结果与讨论}

\section{1. 反应条件优化结果}

我们先前报道了 salen配合物 $\mathbf{C 1}$ 和 $\mathbf{C 2}$ 在以 $\mathrm{H}_{2} \mathrm{SO}_{4}$ 为 共催化剂时可以催化端炔烃的水合反应(表 1 , 实验 1 和 $2)^{[26]}$. 为了更好的解决催化剂和产物的分离问题, 我们 又尝试水溶性钴希夫碱催化剂用于催化炔烃水和反应, 结果表明, 苯乙炔在水溶性 salen-Co(III)配合物 C3 (2\%) 和 $2 \%$ 的 $\mathrm{H}_{2} \mathrm{SO}_{4}$ 的催化下可以顺利转化为相应的苯乙酮 (表 1 , 实验 3 ). 先前的实验表明酸作为共催化剂对炔烃 水合反应影响很大, 我们接着对常见的酸进行了笁选, 如表1中实验3-6所示, 只有盐酸的效果较好, 其它的均 较差, 因此在该催化体系中 $\mathrm{H}_{2} \mathrm{SO}_{4}$ 是最好的共催化剂. 
Naka 课题组 ${ }^{[25]}$ 发现在催化体系中加入二(三氟甲基磺 酰)亚胺时可以促进炔烃的水合反应, 但在我们的催化 体系中二(三氟甲基磺酰)亚胺的催化活性较低. 此外, 当催化体系在惰性气体氛围下, 催化活性明显降低, 甲 醇的作用是不可取代的, 这与Naka课题组 ${ }^{[25]}$ 的结果相一 致.

\section{2. 催化剂循环使用及克级放大实验结果}

在炔烃水合反应中, 产物和催化剂易于分离是磺酸 盐取代的 salen-Co(III)配合物催化剂的优点所在. 反应 结束后, 用 $\mathrm{NaOH}$ 溶液将混合液调到 $\mathrm{pH}=7.0$, 加入少量 水、正已烷或二氯甲烷多次萃取, 有机相和水相分离, 水 溶性的催化剂留在水相中. 蒸发除水, 回收催化剂, 用于 下一次反应. 如表 1 实验 9 所示, 催化剂可以重复使用 3 次, 但其活性略有下降. 接着, 我们做了克级放大实验, 将底物苯乙炔用量扩大 20 倍至 $1.02 \mathrm{~g}$, 反应后苯乙酮分 离收率达96\%(表1, 实验10). 可见, 该催化体系具有一定 的实用性.

\section{3. 底物拓展结果}

为了测试该催化剂的普适性, 在最优反应条件下我 们拓展了底物, 考察了水溶性催化剂 $\mathbf{C} 3$ 对芳环带有不同 取代基的炔烃、多环炔烃、杂环炔烃和脂肪族炔烃等的 催化活性, 结果列于表2中. 可以看出, 芳炔取代基的电 子效应对反应结果影响较大, 供电子基团取代苯炔的转 化率都很高(表 2 , 实验2-8). 在相同条件下, 当芳环上含 有拉电子基团, 如对位上有 $-\mathrm{F},-\mathrm{Cl}$ 和 $-\mathrm{Br}$ 时, 只得到中等 程度的收率; 当我们把 $\mathrm{H}_{2} \mathrm{SO}_{4}$ 用量从 $2.0 \%$ 提高到 $10.0 \%$ 时, 这 3 个炔烃均可以转化为相应的甲基酮 (表 2 , 实验 9-11). 相反, 当苯环上含有强拉电子集团-CN时, 不论
基团在什么位置, 都得不到酮(表 2 , 实验 12). 该催化体 系对多环芳烃和杂环芳烃同样有很好的催化活性(表2, 实验 14和 15), 例如, 4-乙炔基吡啶转化为4-乙酰基吡啶 达分离收率 $98 \%$. 该催化剂对链状炔烃的催化活性较 低, 如癸炔反应后, 只生成37\%的2-癸酮(实验16). 然而, 该催化剂对内炔烃没有催化活性(实验17).

\section{4. 机理探讨}

为了更深入地了解salen-Co(III)配合物催化炔烃水 合的反应机理, 我们选择催化剂 C1催化反应的产物进行 高分辨质谱分析. 如图1所示, 当 $\mathrm{C} 1$ 和苯乙炔在甲醇中 于 $50{ }^{\circ} \mathrm{C}$ 搅拌 $1 \mathrm{~h}$ 后, 在 $M \mathrm{r} / z=325.0379$ 和 449.0652 处出现 两个峰. 其中前者为催化剂峰: [Salen-Co(III) $]^{+}$, 后者推 测是催化剂和苯乙炔三键加成的中间体的峰: [Salen-Co(III)-phenylacetylene $+\mathrm{Na}]^{+}$．再结合文献和我们 之前的工作 ${ }^{[30,34]}$, 推测该反应的机理为: 催化剂活化炔 烃三键进而形成钴配合物炔烃中间体, 然后在酸催化下 甲醇进攻得到烯醚(图式2(1)), 最后在质子酸的作用下 水和甲醇发生了交换生成烯醇, 烯醇快速重排得到甲基 酮(图式2(2)).

\section{4. 结论}

成功地将水溶性 salen-Co(III)配合物用于催化端炔 水合反应, 在使用硫酸为共催化剂的条件下, 大部分端 炔都能高效地转化为甲基酮. 反应后利用萃取的方法即 可使产物与催化剂分离, 避免了操作繁琐的色谱柱分离 方法. 此外, 该催化剂还可以回收重复使用 3 次, 但其活 性略有下降. 综上所述, 该催化体系具有很好的实用性, 其适用性研究正在进行中. 